\title{
Ergonomic concepts in dental medicine
}

\author{
Irina Adriana BEURAN ${ }^{1}$, Augustin $\mathrm{MIHAI}^{1}$, Viorel Stefan PERIEANU ${ }^{1}$, Ileana IONESCU ${ }^{1}$, \\ Oana EFTENE ${ }^{1}$, Simion Gh. DUMITRU ${ }^{2}$, Claudia-Camelia BURCEA ${ }^{1}$, Iuliana BABIUC ${ }^{1}$, \\ Corina Marilena CRISTACHE ${ }^{1}$, Mihai BURLIBASA ${ }^{1}$, Liliana BURLIBASA ${ }^{3}$, Cosmin MEDAR ${ }^{1}$, \\ Maria Glencora COSTACHE ${ }^{1}$, Madalina Violeta PERIEANU ${ }^{1}$, Gabriela TANASE ${ }^{1}$, \\ Oana-Cella ANDREI ${ }^{1}$
}

1“Carol Davila” University of Medicine and Pharmacy, Bucharest, Romania

${ }^{2}$ Dental Practice, Brasov, Romania

${ }^{3}$ Faculty of Biology, University of Bucharest, Romania

\section{ABSTRACT}

Dental medicine is an extremely complex medical field, comprising several specialties (orthodontics and dentofacial orthopedics, prosthodontics, dental surgery, endodontics etc.), which in most cases also require the assistance of a dental nurse.

Purpose. In this paper, we have tried to present a number of very interesting ergonomic aspects in the dental office, which aim at simplifying work at this level.

Material and method. This study was conducted between May and July 2018 using a questionaire applied to a number of 69 dental practitioners from Bucharest.

Results and discussions. Results obtained in this study give us an image about how current dental practitioners understand ergonomics in daily practice.

Conlclusions. All the aspects discussed in this study have an important role in the ergonomy of daily activity of a dental office. More important is that the majority of dentists involved in the stuty understand and apply the ergonomic concepts underlined by this study.
\end{abstract}

Keywords: ergonomics, orthodontics and dentofacial orthopedics, prosthodontics, endodontics

\section{INTRODUCTION}

Ergonomics is a science that is applicable in all fields, including medical and dental medicine.

Ergonomics is actually the scientific discipline that studies the interaction between people and other elements of a system, as well as the profession that applies theories, principles, information and design methods to optimize human activity and the performance of the system it belongs to (definition adopted in August 2000 by the Council of the International Ergonomics Association) (1-4).

Thus, in dental medicine, it is known that the nature of work can determine over time a very high degree of stress and fatigue for the dentist. He must maintain a high degree of concentration 
throughout a working day to be sure of the quality of his work.

In addition, a good dental practitioner is usually rated according to the wide range of maneuvers he/she performs, maneuvers with different degrees of difficulty that test the skill of the practitioner (1-4).

In addition to this, there is a need to set up patient appointments and dental office management, things seemingly simple, but which can also make to the owner of the dental office, who is also a practitioner, a very important stress, along with an extremely stressful state of fatigue (1-8).

And so, from the desire to simplify labor, it was introduced in dental ergonomics, the concept of "dental medicine at four hands" (both the dentist's hands and the nurse are used to the maximum capacity for patient care).

This "4-hand" working method is extremely effective nowadays because it increases the productivity in the dental office, reduces the stress and fatigue of the practitioner, but also represents a good division of labor (1-4).

\section{Purpose of the study}

Dentistry of the 21st Century uses several ideas and theories, most of which come from the past half of the last century (20th century) and which at this time have taken over, developed and processed a multitude of new information on which we will insist especially in this study.

This is especially about the idea of "four-handed dentistry", a modern concept that is supported by several principles, as follows (1-4, 9-13):

- Work strictly in a sitting position;

- Mainly uses the skills of the auxiliary staff of the dental office (nurse responsible for the cabinet, front desk staff) who has been trained in this regard;

- Each component of the practice is organized;

- Visibly simplify the tasks of each participant involved in dental treatment.

These four principles will be explained very simply and concisely in the following (1-4, 15-18):

1. Performing dental maneuvers in seating position substantially reduces fatigue and stress for the medical team, working in the dental office (dentist, nurse).

2. Using the skills of the auxiliary staff in the dentist's office is essential in managing a dentist's time: increases efficiency and productivity.

3. The management of a dental office is a continuous process, which is carried out continuously, like a mobile perpetuum (imaginary technical mechanism that would be able to function continuously, produce energy or perform a mechanical work without receiving energy from the outside) (5). In a modern dental office, there should also be a medical imaging service with specialized staff, which would considerably improve this type of medical unit. An essential role in the dental office is held by the medical team (as known as therapeutic team, surgical team) made up of a dentist and a nurse, which mainly deals with the cabinet itself (including the imaging service where applicable) and also with the methods of performing dental treatments (1-4, 19-22):

- The content and arrangement of the dental office;

- Timely planning of treatment to provide effective specialist services;

- Standardization of usual maneuvers, connected with the design of certain work protocols, which allows the medical team to perform the specific activity in already known sequences;

- Placing the instrumentation in a specific order that is consistent with the protocol sequence previously established.

4. Simplifying work is a certain process by which the simplest maneuver is discovered to perform a well-defined activity. Specifically, over the course of time four classical mechanisms have been established, whereby the activity in this extremely important medical specialty is much easier (1-4):

- Rearrangement (the equipment, the instrument and the specialized materials will be placed in a favorable relationship with the dental team);

- Elimination (an assessment of the maneuvers, systems and movements necessary to carry out the dental work in the cabinet is done, eliminating unnecessary maneuvers and movements, which additionally and unnecessarily adds to this medical activity);

- Combining (eg using a particular instrument or maneuver for multiple purposes);

- Simplification (the idea of simplification is to minimize the number of variables, from all points of view of dental practice, it is actually a process that wants to advance the habits in the labor process).

Thus, the purpose of this material is to make the dentist aware of the usefulness of modern principles in dentistry, in which case we discuss the concept of "4-hand dentistry”. 
The advantages of acquiring this principle, which has already become classic in most of the developed countries of the world from Europe, Asia and America, presents a multitude of advantages, of which we will mention only a few that seemed to us to be the most important: standardized; the correct and efficient placement of the patient as well as the medical team working in the dental office (dentist, nurse); a „honest” organization of time, etc. $(6-8,19-22)$.

\section{MATERIAL AND METHOD}

In order to accomplish this study, we opted for the classical method of the questionnaire. In this regard, I designed a total of seven questions, we consider very concise, regarding this very ergonomic principle of dental practice, namely "4-hand dentistry".

This questionnaire was applied to a number of 69 dental practitioners from Bucharest, the study being conducted between May and July 2018. The subjects included in the study were doctors with at least 5 years of actual dental clinic activity that only operated in a private system, aged between 32 and 63 years.

Female subjects present in this study were 47 (representing 68.12\%), while male subjects were 22 (representing 31.88\%) (Fig. 1).

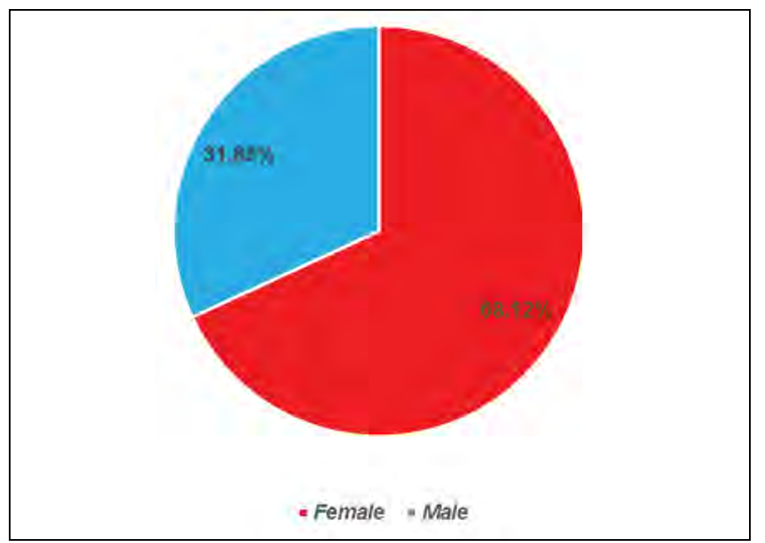

FIGURE 1. Gender distribution of the study group

The questionnaire applied to the 69 subjects on the most important aspects of the ergonomic concept in dentistry, namely "4-hand dentistry", is the following:

\section{Questionnaire}

1. How do you assess the necessity for a dental nurse in achieving therapeutic dental maneuvers? a. Indispensable, even if it requires additional financial effort; $b$. Useful, but not always the financial effort is justified; c. There is no justification for the financial effort to have a cabinet dental nurse.
2. How do you assess the therapeutic maneuvers in dentistry realized in sitting position? a. An extremely important aspect in the ergonomics of the dental office; $b$. An element somewhat helpful in the ergonomics of the dental office; $c$. A useless and time consuming element in daily dental office work.

3. Do you consider that the standardization of the usual procedures of general dentistry, the development of working protocols are: a. An indispensable method in dental office? b. Only a method to improve the efficiency of daily work in a dental office? c. An unnecessary activity, however is part of daily routine of hired personnel?

4. How do you think instrument kits used in daily dental practice should be?: a. To include as many tools as possible to cover as many maneuvers as possible; b. Be as few instruments as possible, but with multiple uses for various maneuvers; $c$. Do the patient only 1-2 dental maneuvers in that session, having only the strict instruments for those maneuvers, being in fact the follower of shorter but frequent treatment sessions.

5. The dental office must have a configuration that?: a. The medical team (dentist, dental nurse) must have access and visibility during all therapeutic maneuvers, while maintaining increased comfort throughout a work day; $b$. Even if space is restricted, the medical team must have as many instruments, equipment and tools as possible, superior endowment being preferred to the expense of the movement space; $c$. It does not matter the size of the workspace, the endowment, it is essential to be able to carry out the dental activity.

6. How do you think the instruments should be delivered to the dentist by the dental nurse? a. The dental nurse transports the instruments required to perform the dental treatment to the dentist's hand in the vicinity of the patient's oral cavity; $b$. The dental nurse removes from the sterile boxes, at the request of the dental practitioner, the instruments necessary for the therapeutic act and puts it on the dental unit's table so that the practitioner uses strictly what he considers necessary; $c$. The dentist is supposed to bring himself on the dental unit's table, the necessary instrument for performing the dental treatment.

7. How do you assess the time management in general dentistry that you do daily? a. An indispensable element in the ergonomics of the dental office; $b$. A relative factor in the ergonomics of the dental office; c. An irrelevant item, the dental offices day-to-day work being carried out very efficiently, even without giving much importance to time management. 


\section{RESULTS AND DISCUSSION}

The answers obtained by applying the questionnaire were centralized and organized as follows:

The presence and involvement of dental nurse in the achievement of treatment is seen differently by the participants in the study: 42 of them (representing 60.87\%) consider it indispensable, 22 participants (representing $31.88 \%$ ) consider it useful, and while only 5 participants (representing $7.25 \%$ ) consider it a financial effort that is not justified (Fig. 2).

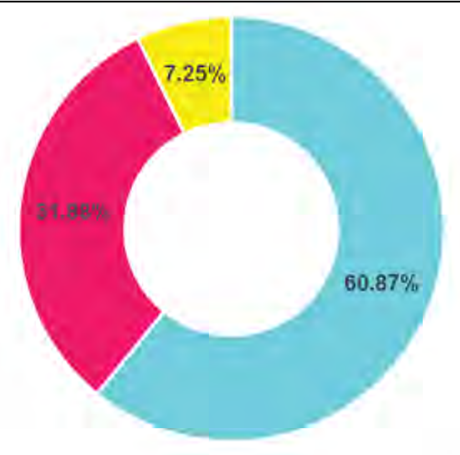

-Indinspensable - Useful - Not justified

FIGURE 2. The utility of dental nurse in the achievement of therapeutic acts

Achieving therapeutic acts in sitting position is considered as an extremely important action in the ergonomics of the dental office activities by 56 of the study respondents (81.16\%). The rest of the subjects consider this aspect to be of most helpful (10 subjects $-14.49 \%$ ) or even an unnecessary and time-consuming method (3 subjects - 4.35\%) (Fig. 3).

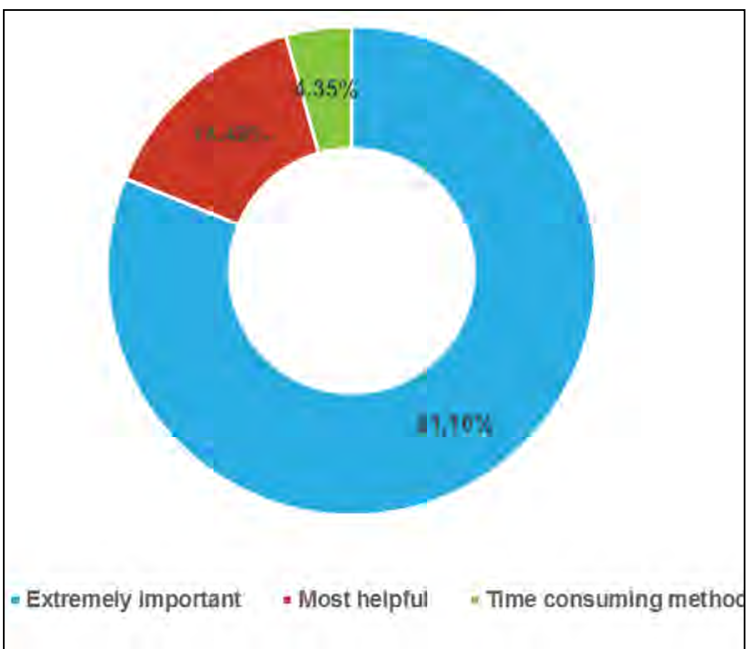

FIGURE 3. Doctors' attitudes towards working in a sitting position
Of the interviewed subjects, 60 (86.96\%) considered working protocols to be an indispensable method in standardizing the usual procedures, while only 9 subjects (13.04\%) considered this as a way of improving the efficiency of daily activities (Fig. 4).

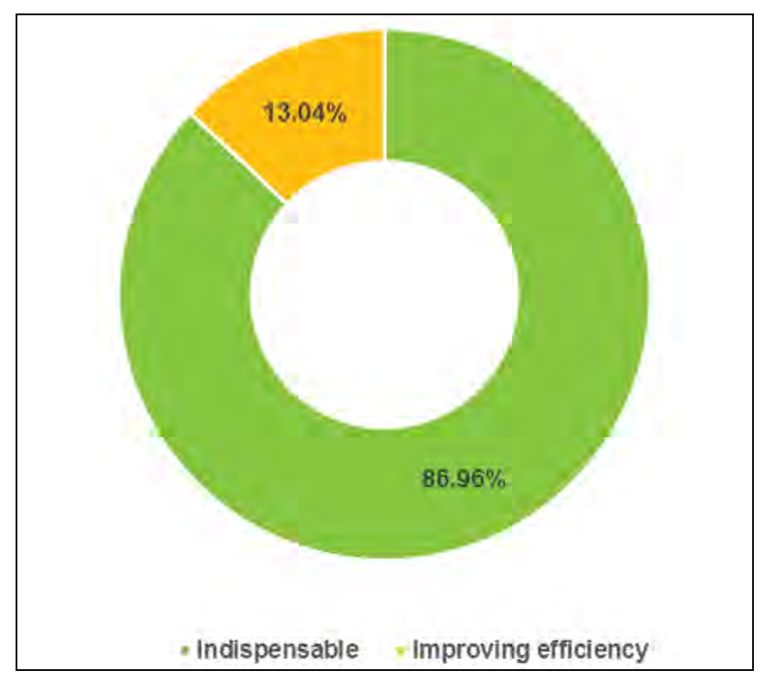

FIGURE 4. The need to implement working protocols

Regarding the composition of instrument kits used in daily practice, the results were predictable, more than half of the respondents (42 - representing $60.87 \%$ ) considered a small number of multiple use instruments to be necessary, 20 of the respondents (representing 28,99\%) prefer as many tools as possible, while a small number of respondents ( 7 - representing $10.14 \%)$ prefer that the kits contain the instruments needed for the treatment scheduled for that treatment session (Fig. 5).

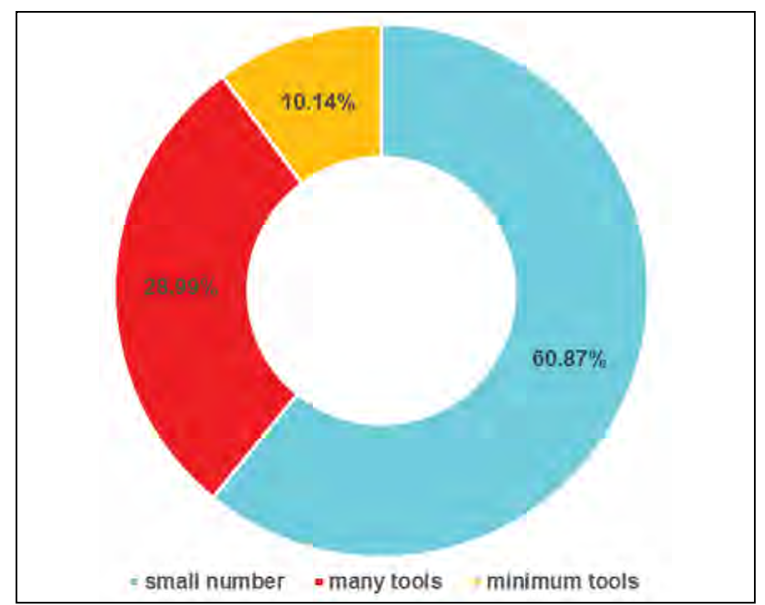

FIGURE 5. Composition of instrument kits used in current practice

The configuration of the dental office is another aspect of this study. Thus, most practitioners (52 - representing 75.36\%) consider that they 
must have access and visibility during all therapeutic maneuvers, 15 doctors $(21.74 \%)$ consider that they should have as many devices as possible and only two doctors considers that the size of the workspace is irrelevant (Fig. 6).

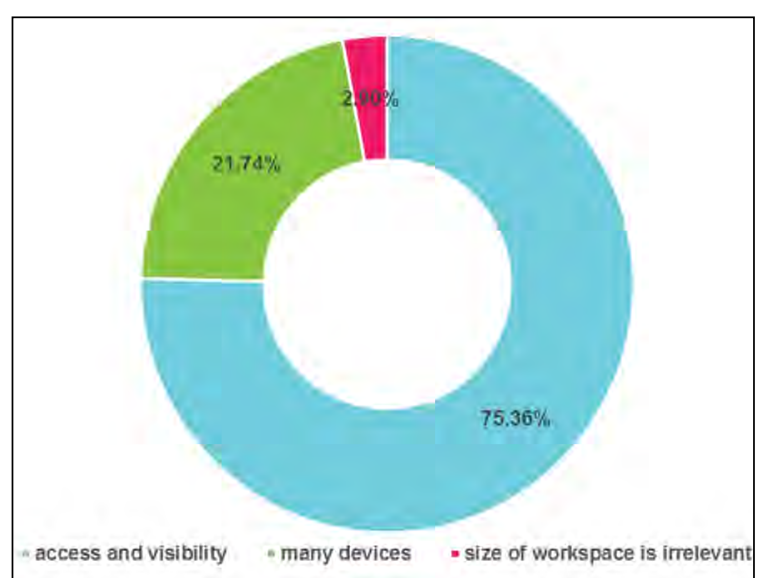

FIGURE 6. Configuration of the working space in the dental office

Handling the instruments during the therapeutic procedures, another organization part of the activity in the dental office, gives divided opinions to the practitioners involved in the study. 33 of the them $(47.83 \%)$ believes that the nurse must make available to the physician the necessary instruments, 27 of the practitioners (39.13\%) consider that the nurse should place the instrument on the dental unit's table and the doctor should choose the appropriate tools for the current maneuvers. Only 9 practitioners (13.04\%) believe that the doctor should be the one who prepares the necessary instruments (Fig. 7).

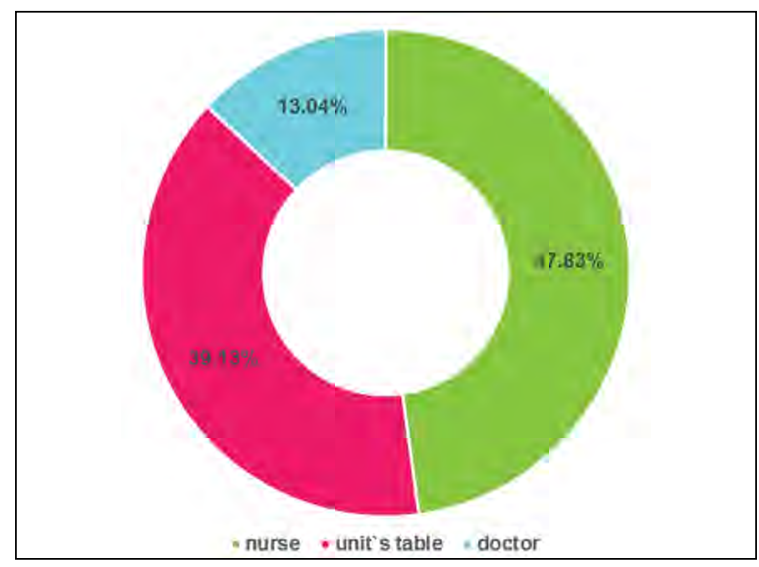

FIGURE 7. Preparing the needed instruments for current maneuvers

The last aspect of this study relates to time management in the current work of a dental office. It is to be appreciated that the vast majority of practitioners (65 representing 94.20\%) consider this aspect as an indispensable element in the ergonomics of the cabinet's work, while only 4 practitioners (representing 5.80\%) consider this aspect only as a relative factor (Fig. 8).

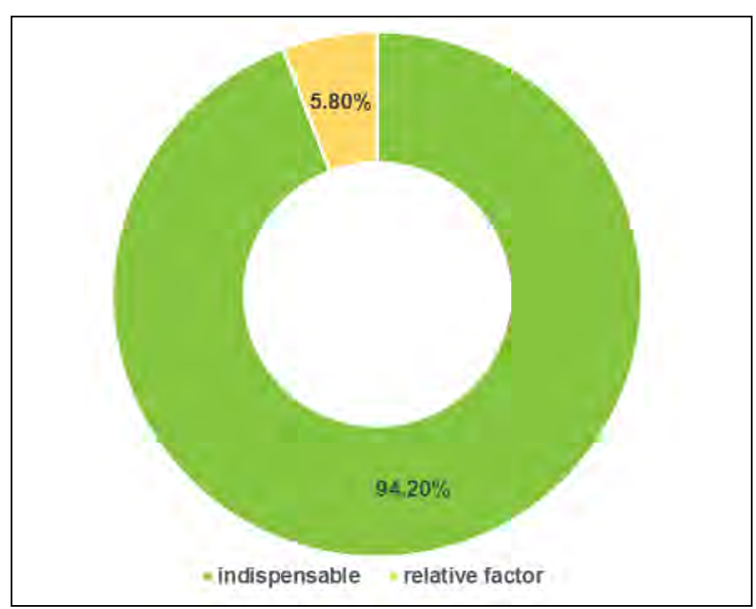

FIGURE 8. Time management in the current work of the dental office

\section{CONCLUSIONS}

Following the application of the questionnaire regarding the principle of "4-hand dentistry" and the study of the answers of the 69 dental practitioners, on this issue, which we consider it to be extremely interesting to, we can conclude the following:

Constructive attitude within the medical team working in the dental office. There must be more than constructive collaboration between the dentist and dental nurse. Dental nurse needs to get to that routine, in which she will predict the wishes of the dentist with which she collaborates. If in time there is no such attitude in the dental medical team, we will definitely have an increased rate of failure.

Favorable work environment. In order to be able to apply this "4-handed dentistry" principle in a dental office, the configuration used in the cabinet design should allow the medical team (dentist, nurse) to have access and visibility during all therapeutic maneuvers, while maintaining increased comfort throughout a day's work.

A proper setting for both the patient and the medical team working in the dental office. Studies conducted over time have highlighted the most favorable positions of both patients and the medical team working in dental office (dentist, nurse) to work effectively in different segments of the oral cavity. But these special points will be highlighted and described in another material.

Standardized operating procedures. Most procedures in dental practice are almost always the 
same, so it requires minimal variation. Thus, the most frequent therapies can be standardized so that the medical team working in dental office (dentist, nurse) can perform them with maximum efficiency after a pre-established protocol.

Simplified equipment and instruments. The goal is to reduce the equipment and the number of instruments to a minimum, by selecting the ones that are used for a specific procedure.

Efficient delivery of instruments. One of the standard methods of transferring the instrument without making unnecessary, time-consuming movements is where the dental nurse carries the items necessary to perform the dental treatment act to the dentist's hand in the vicinity of the patient's oral cavity.

A good time organization. Time management is actually one of the most important items in a dental office. Specifically, a good organization of time gives the medical team working in the dental office (dentist, dental nurse) the opportunity to offer the best choices for an effective dental treatment for a maximum number of patients.

\section{Acknowledgement}

In this article, all the authors have equal contributions with the first author.

\section{Conflict of interest: none declared} Financial support: none declared

\section{REFERENCES}

1. Ergonomos. Ergonomos. Ergonomos Solutii Ergonomice Profesionale. [Interactiv] [Cited: 20 February 2019.] https://www. ergonomos.ro.

2. Burlibaşa M. Noţiuni generale de management, organizare ergonomică şi protecţia muncii în laboratorul de tehnică dentară. Editura Ars Docendi, Bucureşti, 2005.

3. Uram-Tuculescu S. Instrumente, dispozitive şi aparate în laboratorul de tehnică dentară. Editura Helicon, Timişoara, 1998.

4. Miyasaki-Ching CM. Elemente clinice de stomatologie. Editura All Educational, București, 2001.

5. Dexonline. Perpetuum mobile. dexonline.ro. [Interactiv] [Cited: 20 February 2019.] https://dexonline.ro/definitie/perpetuum $\% 20$ mobile.

6. Gall II. Asistența stomatologică. Editura Didactică și Pedagogică, București, 1971.

7. Rosenstiel SF, Land MF, Fujimoto J. Contemporary fixed prosthodontics. Fourth Edition. Mosby Elesvier, 2006.

8. Anusavice K. Phillip's science of dental materials. 11th edn. Philadelphia : WB Saunders, 2003.

9. Gavrila L, Mircea L. Chromatin and chromosomal fine structure in spermatogenesis of some species of amphibians. Zygote. 2001; 9(3), 183-192.

10. Cristache CM, Burlibașa M, Cristache $\mathrm{G}$ et al. Zirconia and its biomedical applications. Metalurgia International. 2011; 16(7), 18, p. 18-23.
11. Burlibașa M, Cernușcă-Mițariu M, Burcea $\mathrm{CC}$ et al. Halogen compounds - theoretical, physiological and practical aspects regarding the decontamination, disinfection and sterilisation of intsruments and biomaterials in dental medicine practice. Metalurgia International. 2013; Vol. XVIII, Spec. Issue No. 3, p. 54-57.

12. Tănase $G$, Burlibașa $M$, Muntianu L et al. Testing the antibacterial potential of biomaterials in medical practice. Metalurgia International. 2009; Vol. XV, Spec. Issue No. 2, p. 160-162.

13. Burlibașa M, Muntianu L, Tănase $\mathrm{G}$ el al. Study on microbial contamination of biomaterials in medical practice. Metalurgia International. 2009; Vol. XV, Spec. Issue No. 2, p. 163-166.

14. Popescu FD, Popescu SM, Burlibașa M. Platinum group metals a contact allergens in oral rehabilitation. Metalurgia International. 2009, Vol. XV, Spec. Issue No. 3, p. $12-18$.

15. Cristache $\mathrm{CM}$, Ionescu $\mathrm{C}$, Cristache $\mathrm{G}$ et al A 5-year prospective randomised clinical trial on the efficiency of two different attachement systems as retention for implant-supported mandibular overdenture. Radiographic assesment, cost analysis and final evaluation of treatment $s$ success. Metalurgia International. 2008; Vol. XIV, Spec. Issue No. 16, p. 27-34.

16. Burlibașa $M$, Cuculescu $M$, Tănase $G$ et al. Dental alloys of prothetic use - a retrospective of their use in Romania.
Metalurgia International. 2008; Vol. XIV, Spec. Issue No. 16, p. 51-53.

17. Cristache $\mathrm{CM}$, lonescu $\mathrm{C}$, Burlibașa $\mathrm{M}$ et al. Rettentive anchors versus magnets as attachment systems for mandibular overdenture. A 5 year prospective randomised clinical study. Metalurgia International. 2009; Vol. XIV, Spec. Issue No. 16, p. $59-64$.

18. Burlibașa $L$, Zarnescu O. In vivo effects of Trichostatin A - A histone deacetylase inhibitor-On chromatin remodeling during Triturus cristatus spermatogenesis. Animal reproduction science. 2013; 142 (1-2), 89-99.

19. Burlibașa L, Gavrila L. Developmental epigenetics: roles in embryonic development, in Nutrition in Epigenetics (eds. Niculescu MD, Haggarty P); 2011, Ch. 6, p. 105-126, Willey-Blackwell Publishing.

20. Ștețiu AA, Oleksik M, Oleksik V et al. Mechanical behavior of composite materials for dental obturations. Romanian Biotechnological Letters. 2013; 18(4), p. 8528-8538.

21. Mocuța D, Popovici LR, Dumitriu AS et al. Life quality-condition of social welfare. Metalurgia International 14, 2009, 62-64.

22. Burlibașa M, Burlibașa L, Gavrilă LB et al. microRNA a macro Revolution in Medical Biotechnologies. Romanian Biotechnological Letters 13(6), 2008, 3977-3983. 